\title{
Normes de densité pour les hêtraies du Nord-Est et du Nord-Ouest de la France $\left({ }^{1}\right)$
}

\author{
N. LE GOFF ef J.-M. OTTORINI \\ avec la collaboration technique de L. GARROS et M. RAVART \\ Station de Sylviculture et de Production, \\ Centre national de Recherches forestières, I.N.R.A., \\ Champenoux, 54280 Seichamps
}

\begin{abstract}
Résumé
Depuis 1961 le «facteur de concurrence des cimes » ou « CCF » de Krajicek et al. - facteur relié très étroitement au «TAR » de Chisman et Schumacher - a prouvé son efficacité en tant que mesure objective de la densité d'un peuplement à travers de nombreuses applications aux U.S.A.. Le présent travail s'inspire d'un article publié par S. F. Gingrich sur « la densité et des normes de densité pour des peuplements feuillus » aux U.S.A. ; ici, la densité maintenue constante de peuplements de hêtre régulièrement éclaircis est utilisée comme densité de référence par l'intermédiaire de la relation liant le diamètre de la couronne des arbres à leur diamètre à $1,30 \mathrm{~m}$.

Deux régions de croissance pour le hêtre ont été distinguées dans le nord de la France ; un échantillonnage aléatoire de peuplements de hêtre de différents âges dans les deux meilleures classes de fertilité de chaque région a montré l'existence d'une bonne relation linéaire entre le diamètre de la couronne des arbres et leur diamètre à 1,30 m pour les peuplements du nord-est d'une part, et ceux du nord-ovest d'autre part. D'après les études de Curtin et de Gingrich, cette relation caractéristique permet de s'assurer d'une densité constante des peuplements échantillonnés et de construire alors une mesure de densité à la manière de Krajicek et al.

Une attention particulière s'est révélée nécessaire dans l'ajustement des relations pour obtenir des résultats cohérents dans l'établissement des normes de densité pour chaque région. Ces normes définissent les caractéristiques (surface terrière et nombre de tiges à l'hectare, diamètre moyen) que doivent avoir les peuplements de hêtre pour croître avec une densité constante, exprimée en pourcentage de la densité moyenne observée actuellement dans les peuplements éclaircis de façon habituelle dans le nord de la France.
\end{abstract}

\section{Introduction}

La sylviculture des hêtraies en France a pour but l'obtention de futaies régulières à croissance relativement rapide et composées d'arbres de haute qualité. Le travail présenté ici a été entrepris pour répondre au besoin de disposer de "normes " pouvant servir à guider cette sylviculture dans les grandes hêtraies du nord de la France.

(1) Les données utilisées pour cette étude ont été recueillies sur le terrain suivant un protocole de J. Bouchon interprété et mis en application par M. Ravart et L. Garros ; tous les trois sont respectivement chargé de recherche eftechniciens d̀ la Station de Sylviculture et de Production. 
Les normes proposées définissent les caractéristiques que doivent posséder les peuplements pour avoir constamment une «densité » donnée, dans le but d'aider à contrôler leur croissance et leur développement au moyen d'éclaircies. Pour obtenir ces normes une attention particulière a été accordée à l'évaluation des conditions de croissance des arbres en peuplement du point de vue de l'espace vital individuel, de façon à définir une mesure de densité suivant les principes du «Crown Competition Factor » ou «CCF » de Krajicek et al. (1961).

L'étude réalisée s'inspire d'un article de Samuel, F. Gingrich (1967). Sestravaux ont pu être adaptés malgré la nature différente des peuplements de nos régions qui sont tous soumis à des traitements sylvicoles divers et réguliers depuis leur plus jeune âge.

Après quelques rappels concernant la notion de densité de peuplement, les deux régions d'étude (nord-est et nord-ouest de la France) seront caractérisées vis-à-vis de la croissance du hêtre. Les chapitres suivants marqueront les étapes successives qu'il a fallu franchir pour établir les normes de densité des peuplements de hêtre dans ces deux régions.

\section{I. - Rappels sur la notion de densité de peuplement}

La densité d'un peuplement forestier concerne les conditions de croissance des arbres ayant trait à leur concurrence. C'est une notion difficile à formuler et les nombreux efforts pour l'exprimer quantitativement de façon adéquate correspondent à un besoin de progrès dans l'étude de la croissance des peuplements forestiers et dans les pratiques sylvicoles.

Malgré les récents travaux de Drew et Flewelling (1977) qui ont procédé par complète analogie avec les méthodes utilisées en agronomie dans l'étude des plantes annuelles (voir l'analyse de Willey et Heath, 1969), il vaut mieux considérer comme Curtis (1970) que les arbres posent un problème différent parce que leurs exigences vis-à-vis du milieu ambiant évoluent considérablement avec l'âge. Dès 1928, le « Facteur d'Espacement» de Hart (Hummel, 1954), tenait compte de cette particularité, mais de façon un peu arbitraire, et selon un principe d' « espacement moyen par arbre » (Pardé, 1961) qui peut être trop synthétique. Parmi les nombreuses mesures de densité apparues depuis, certaines proposent de tenir compte avec plus de réalisme de la place disponible pour chaque individu d'un peuplement, relativement à ses besoins. Une étude bibliographique (Ottorini, 1978) a eu pour but de montrer la valeur de ces mesures de densité, en particulier : le CCF de Krajicek et al., et le «Tree Area Ratio » ou «TAR» de Chisman et Shumacher (1940).

Ces deux mesures de densité sont basées sur la relation linéaire - indépendante de l'âge et de la station - qu'il y a entre le diamètre de cime et le diamètre à $1,30 \mathrm{~m}$ des arbres de peuplements de densité très faible dans le cas du CCF, ou au contraire extrêmement forte dans le cas du TAR. Gingrich (1967) a montré que ces mesures de densité étaient proportionnelles, et donc équivalentes $\left({ }^{1}\right)$. Indépendamment, Curtin (1970) a pu observer que d'une façon plus générale la relation diamètre de cimediamètre à 1,30 m caractérisait une densité de peuplement $\left({ }^{2}\right)$ et que les droites corres-

(1) Avec un noyer (Carya ovata) et deux variétés de Chênes (Quercus alba, Quercus velutina).

$\left({ }^{2}\right)$ Avec un eucalyptus (Eucalyptus obliqua). 
pondant aux diverses conditions de densité étaient homothétiques (fig. 1). Son étude confirme donc les observations de Gingrich et apporte un crédit supplémentaire aux principes que ce dernier a utilisés.

Tout ce qui suit fera largement appel à ces faits expérimentaux et à leurs conséquences dans la définition d'une mesure de densité de peuplement.

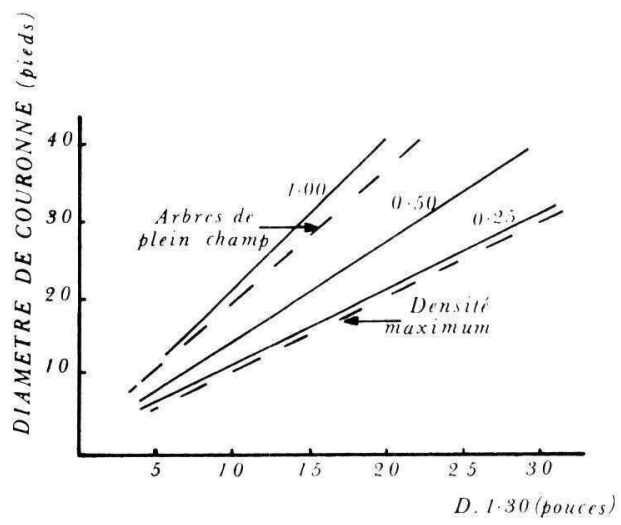

FIG. 1. - Relations caractéristiques de différentes densités de peuplement pour une espèce donnée. Les droites en trait plein correspondent à trois statuts concurrentiels théoriques des arbres (évalué par le rapport de la hauteur de cime à la hauteur totale). Les droites en trait discontinu correspondent à des conditions réellement observées (d'après Curtin).

Relationship between crown width and diameter for trees of a given species under specified conditions of competition status (measured by the crown length to height ratio) (redrawn from Curtin).

\section{II. - Régions et stations}

Les grandes hêtraies du nord de la France recouvrent des régions aux climats assez différenciés allant d'un climat tempéré océanique dans le nord-ouest du Bassin pari-

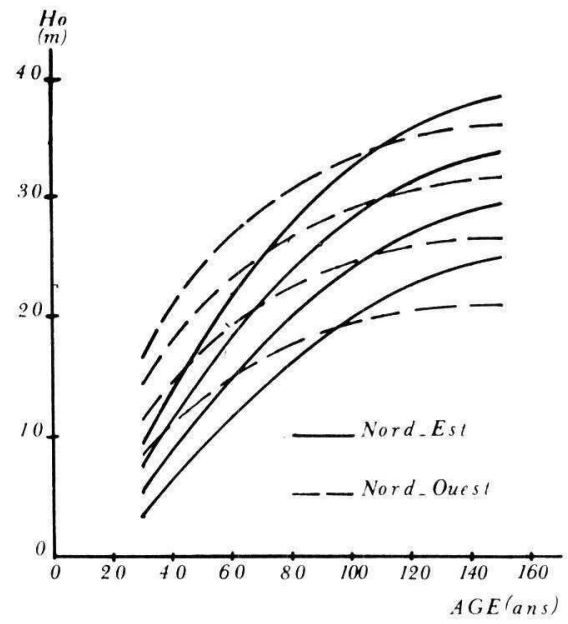

FIG. 2. - Courbes de croissance en hauteur dans le nord-est et dans le nord-ouest de la France.

Height growth curves in the north-east and the north-west of France. 
sien à un climat de type plus continental dans le nord-est de la France. Un travail antérieur (Le Goff, 1974) basé sur la comparaison de courbes de croissance en hauteur a montré que deux régions climatiques pour la croissance du hêtre pouvaient être distinguées dans cette partie nord de la France : le nord-ouest du Bassin parisien, d'une part, où la croissance en hauteur est tout à fait semblable à celle observée en Angleterre, le nord-est de la France y compris le nord-est du Bassin parisien, d'autre part, où la croissance en hauteur se rapproche davantage de celle observée en Allemagne ( ${ }^{1}$ ). La figure 2 permet de comparer les allures respectives de la croissance en hauteur dans ces deux régions. Les courbes tirées de la table de production de Schober (1971) pour les différentes classes de fertilité s'appliquent au nord-est, celles de Hamilton et Christie (1971) au nord-ouest.

Seules les bonnes stations des hêtraies étudiées dans chaque région climatique ont été retenues ; les peuplements croissant dans ces conditions peuvent être avantageusement soumis à une sylviculture intensive (éclaircies fortes) et fournir alors les bois de haute qualité. Les peuplements échantillonnés se situent donc au niveau des deux meilleures classes de fertilité de la table de production allemande pour les hêtraies du nord-est d'une part (fig. 3A), de lo table de production anglaise pour les hêtraies du nord-ouest d'autre part (fig. 3B).
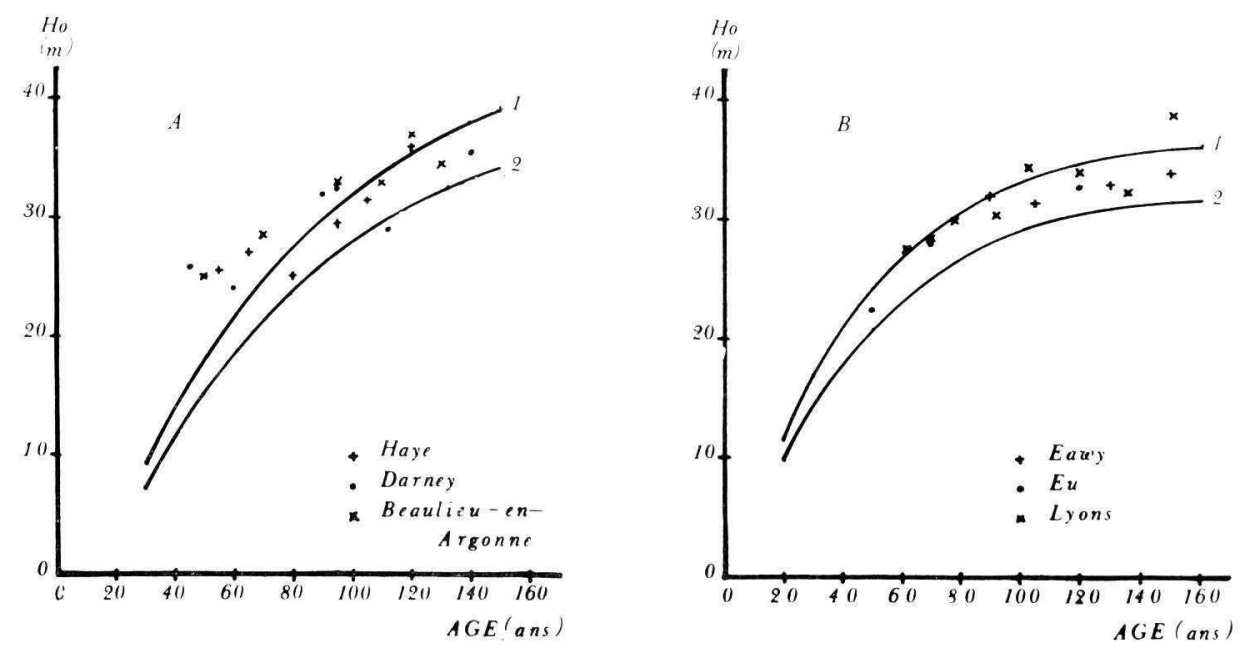

FIG. 3A. - Couples hauteur dominante-âge des placettes du nord-est et courbes moyennes de croissance en hauteur pour les classes de fertilité 1 et 2 dans le N.-E.

FIG. 3B. - Couples hauteur dominante-âge des placettes du nord-ouest ef courbes moyennes de croissance en hauteur pour les classes de fertilité 1 ef 2 dans le N.-O.

Dominant height-age datas for the sampled stands in the north-east (A) and the north-west (B) of France and mean height growth curves for site quality classes 1 and 2 in each region.

(1) Ces deux régions climatiques se trouvent caractérisées par des valeurs différentes de l'indice C. V. P. de Paterson-Parde (I) : pour le nord-ouest du Bassin parisien $300<1<399$, pour le nord-est de la France $200<1<299$ (voir Parde, 1959). 


\section{III. - Recueil et analyse des données}

\section{A. - Données recueillies}

Les peuplements retenus pour cette étude appartiennent tous aux grands massifs domaniaux du nord de la France où le hêtre croît en futaie régulière du fait de traitements sylvicoles suivis. Les hêtraies échantillonnées sont les suivantes :

- dans le nord-est de la France, forêts de Haye en Meurthe-et-Moselle (7 000 ha), de Darney dans les Vosges (5 300 ha), de Beaulieu-en-Argonne dans la Meuse (2 $600 \mathrm{ha})$;

- dans le nord-ouest du Bassin parisien, forêt d'Eawy (6 $600 \mathrm{ha})$, Basse et Haute forêt d'Eu (6 500 ha) en Seine-Maritime, forêt de Lyons dans l'Eure (10 600 ha).

Dans chaque région l'intervalle des âges le plus large possible a été échantillonné (fig. $3 \mathrm{~A}$ et $3 \mathrm{~B}$ ) ( $\left.{ }^{1}\right)$. Pour cela dix-huit placettes ont été installées dans le Nord-Est et quinze placettes dans le Nord-Ouest, choisies de façon aléatoire mais de manière à présenter des conditions de station homogènes et de peuplement pur au couvert fermé.

TABLEAU 1

Age moyen et codage des placettes échantillon réparties en 11 classes d'âge pour les forêts du Nord-Ouest et du Nord-Est

\begin{tabular}{|c|c|c|c|c|c|c|}
\hline \multirow{2}{*}{$\begin{array}{l}\text { Classe } \\
\text { d'âge }\end{array}$} & \multicolumn{3}{|c|}{ Nord-Ouest } & \multicolumn{3}{|c|}{ Nord-Est } \\
\hline & Eawy & Eu & Lyons & Haye & Darney & Beaulieu \\
\hline 1 & & \multirow[t]{2}{*}{$50(301)$} & & $55(701)$ & $45(801)$ & $50(901)$ \\
\hline 2 & & & $62(502)$ & 65 (702) & $60(802)$ & \\
\hline 3 & & \multirow[t]{5}{*}{$70(303)$} & $70(503)$ & & & $70(903)$ \\
\hline 4 & & & 78 (504) & 80 (704) & & \\
\hline 5 & 91 (105) & & $92(505)$ & 95 (705) & $90(805)$ & $95(905)$ \\
\hline 6 & 105 (106) & & $103(506)$ & 105 (706) & $96(806)$ & \\
\hline 7 & & & & & $112(807)$ & $110(907)$ \\
\hline 8 & & \multirow[t]{4}{*}{$120(208)$} & $119(508)$ & $120(708)$ & & $120(908)$ \\
\hline 9 & $130(109)$ & & & & & $130(909)$ \\
\hline 10 & & & $136(510)$ & & $140(810)$ & \\
\hline 11 & 150 (111) & & $151(511)$ & & & \\
\hline
\end{tabular}

Seuls les arbres appartenant à l'étage des dominants et des codominants ont été mesurés. Chaque placette devait contenir environ quarante arbres; les placettes occupent ainsi des superficies variables allant de 4 à 24 ares.

Sur chaque arbre les caractères suivants ont été mesurés :

Diamètre à $1,30 \mathrm{~m}$ mesuré au ruban.

Hauteur totale mesurée au dendromètre Blume-Leiss.

Hauteur de la cime (prise comme hauteur de la masse foliaire) mesurée au BlumeLeiss.

(1) L'âge retenu est un âge moyen, les peuplements n'étant jamais rigoureusement équiennes : la différence d'âge entre les arbres d'un peuplement peut atteindre 20 ou 30 ans. 
Surface de projection de la cime ou surface de couronne (à partir de la mesure de huit rayons de cette projection).

Diamètre de couronne : défini comme le diamètre du cercle de surface égale à celle de la projection de cime.

Les peuplements ont été répartis dans onze classes d'âge, les âges des peuplements d'une même classe différant de moins de 10 ans. Chaque placette a reçu alors un numéro de code, composé à partir d'un code «forêt» et du numéro de la classe d'âge à laquelle elle appartient (tabl. 1).

\section{B. - Analyse des données}

Le rapport de la hauteur de cime à la hauteur totale des arbres permet d'évaluer les effets de la concurrence au niveau individuel et peut constituer une mesure de son
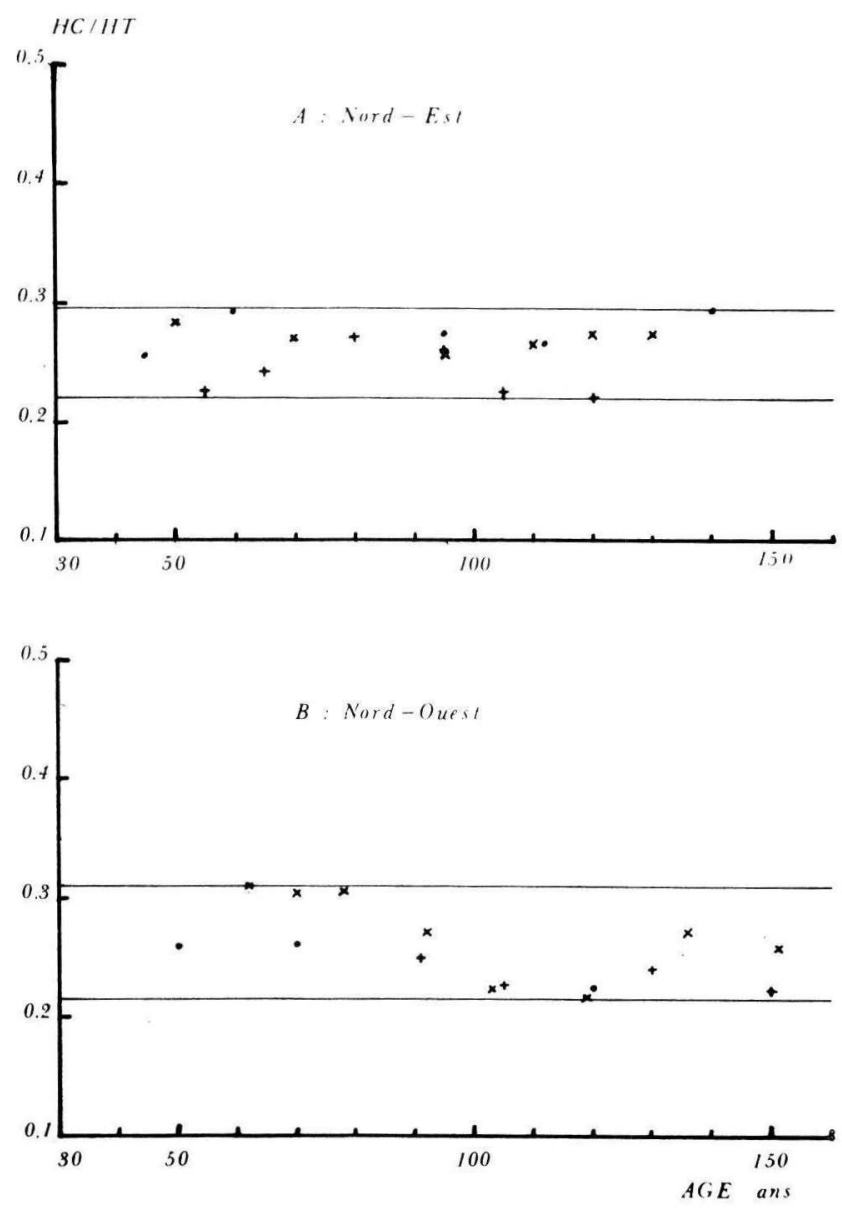

FIG. 4. - Intervalles de variation de la densité des placettes (voir légende de la figure 3) mesurée par le rapport hauteur de cime/hauteur totale dans le N.-E. (A) et dans le N.-O. (B).

Density of the sampled stands measured by the mean crown length to height ratio in the north-east (A) and the north-west (B). 
NORMES DE DENSITÉS POUR LES HÊTRAIES

287

intensité. Ce rapport assez suggestif a été utilisé par Curtin (1970), et la moyenne de ces valeurs pour les arbres d'un peuplement a été préconisée par Czarnowski en 1961 comme mesure de la densité du peuplement. Dans le Nord-Est la densité des peuplements échantillonnés mesurée de cette façon est comprise entre 0,22 et 0,30. Dans le Nord-Ouest elle va de 0,21 à 0,31 . Pour les deux régions, elle est indépendante de l'âge (fig. 4). Comme les données disponibles provenant d'expériences d'éclaircies suivies en France indiquent que la densité ainsi définie peut être voisine de 0,10 pour les peuplements non éclaircis, ou à l'opposé dépasser 0,4, il est permis de penser que la

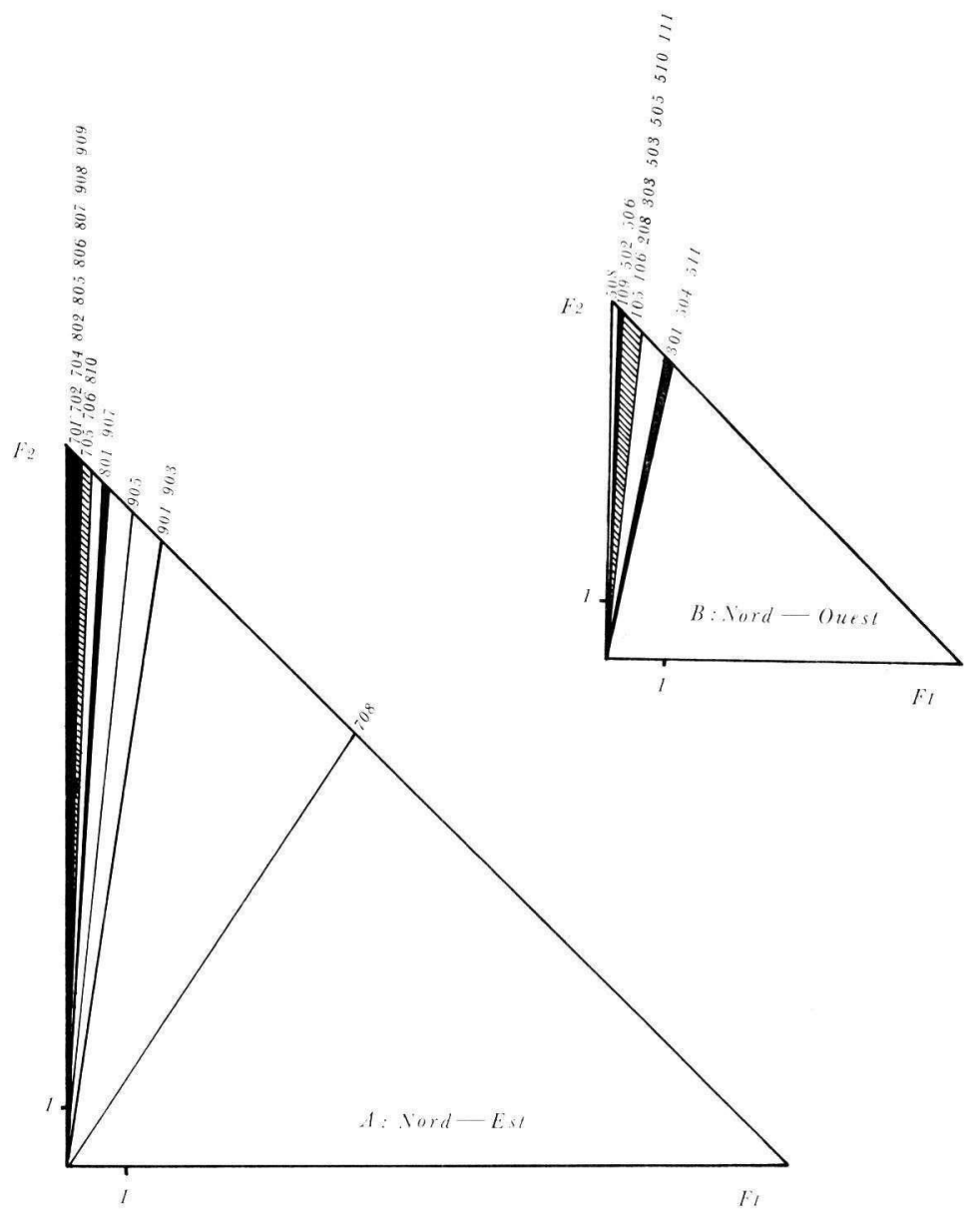

FIG. 5. - Représentation sommaire des écarts entre les droites « diamètre de couronne-diamètre à 1,30 m » ajustées par peuplement et la droite moyenne de référence ajustée pour tous les peuplements d'une même région (A : Nord-Est ; B : Nord-Ouest) (voir le texte pour les détails). Avec une échelle appropriée l'hypothénuse d'un triangle représente la somme totale des écarts ; sur celle-ci, l'écart d'un peuplement particulier à la moyenne peut être apprécié en valeur relative comme contribution à cette somme.

Clustering of the crown width-dbh relationships of the various sampled stands in both regions figured by the differences befween the residual sums of squares relative to the mean line and each particular stand line. Each difference is combined with the total sum of all differences to help appreciate its relative importance. 
densité des peuplements échantillonnés n'est pas très variable. Ceci se trouve confirmé par l'examen des diagrammes de dispersion du diamètre de couronne en fonction du diamètre à 1,30 m pour chacun des peuplements dans les deux régions. Le paragraphe suivant en propose une description.

Les ensembles de points relatifs aux différents peuplements dans chaque région montrent une très nette dépendance linéaire du diamètre de couronne vis-à-vis du diamètre à 1,30 $\mathrm{m}$ et se présentent avec une dispersion homogène. Cette dispersion est à peu près comparable d'un peuplement à l'autre, mais surtout, pour chaque région, les droites qui pourraient représenter chaque ensemble de points sont peu différentes. La figure 5 se propose de représenter sommairement ces différences pour le NordEst et le Nord-Ouest. Dans les deux cas, une «droite moyenne » a été ajustée par les moindres carrés à l'ensemble des données pour servir de référence ; cette droite a été ensuite comparée aux droites déterminées de la même façon par peuplement. Pour chaque peuplement, «l'écart » entre les deux droites concurrentes a été mesuré par la différence entre les sommes de carrés résiduelles se rapportant respectivement à l'une et à l'autre. Sur la figure 5, chaque peuplement est représenté par un vecteur dont l'abscisse $F_{1}$ est égale à l'écart entre la droite de référence et la droite ajustée aux données provenant de ce peuplement. L'ordonnée $F_{2}$ est égale au total des écarts entre la droite de référence et les droites particulières aux autres peuplements. De cette façon, l'échelle de la figure est indifférente pour apprécier l'écart entre chaque droite et la droite moyenne $\left({ }^{1}\right)$. Si l'on tient compte des rappels de la section I, ces faits rejoignent l'hypothèse du précédent paragraphe, relative à la densité des peuplements étudiés.

Enfin, pour compléter et conclure cette analyse, l'examen graphique montre que pour chaque régression du diamètre de couronne en fonction du diamètre à $1,30 \mathrm{~m}$ par peuplement, les résidus de la régression ne présentent aucune dépendance vis-àvis des autres caractères individuels, en particulier la hauteur totale ou le rapport de la hauteur de cime à la hauteur totale.

\section{IV. - Calcul de la relation de base pour chaque région}

La disparité observée dans chaque région entre les relations diamètre de couronne-diamètre à $1,30 \mathrm{~m}$ pour les différents peuplements (fig. 5) n'a pas pu être expliquée de façon effective par des caractéristiques connues des peuplements : une tentative n'a permis de réduire l'écart-type résiduel que de 1 p. 100 pour le Nord-Est et de 6 p. 100 pour le Nord-Ouest $\left({ }^{2}\right)$. Bien que ces deux différences soient statistiquement significatives, il n'en a pas été tenu compte : la correction d'un léger biais par l'introduction d'une nouvelle variable explicative risquait de se faire aux dépens de la fidélité des estimations. Pour chaque région, l'ensemble des données a donc été confondu et considéré comme provenant d'arbres se trouvant dans les mêmes conditions de densité ; seul le peuplement 708 de la région nord-est, qui s'était individualisé dans l'analyse précédente, a été écarté.

(1) Cette figure prétend seulement exposer certaines caractéristiques d'un ensemble de représentations graphiques trop nombreuses pour être reproduites ; elle n'a « a priori » pas réellement vocation de méthode statistique : sa lecture devrait se faire de façon informelle.

${ }^{2}$ ) En utilisant le diamètre moyen de chaque peuplement comme co-variable. 


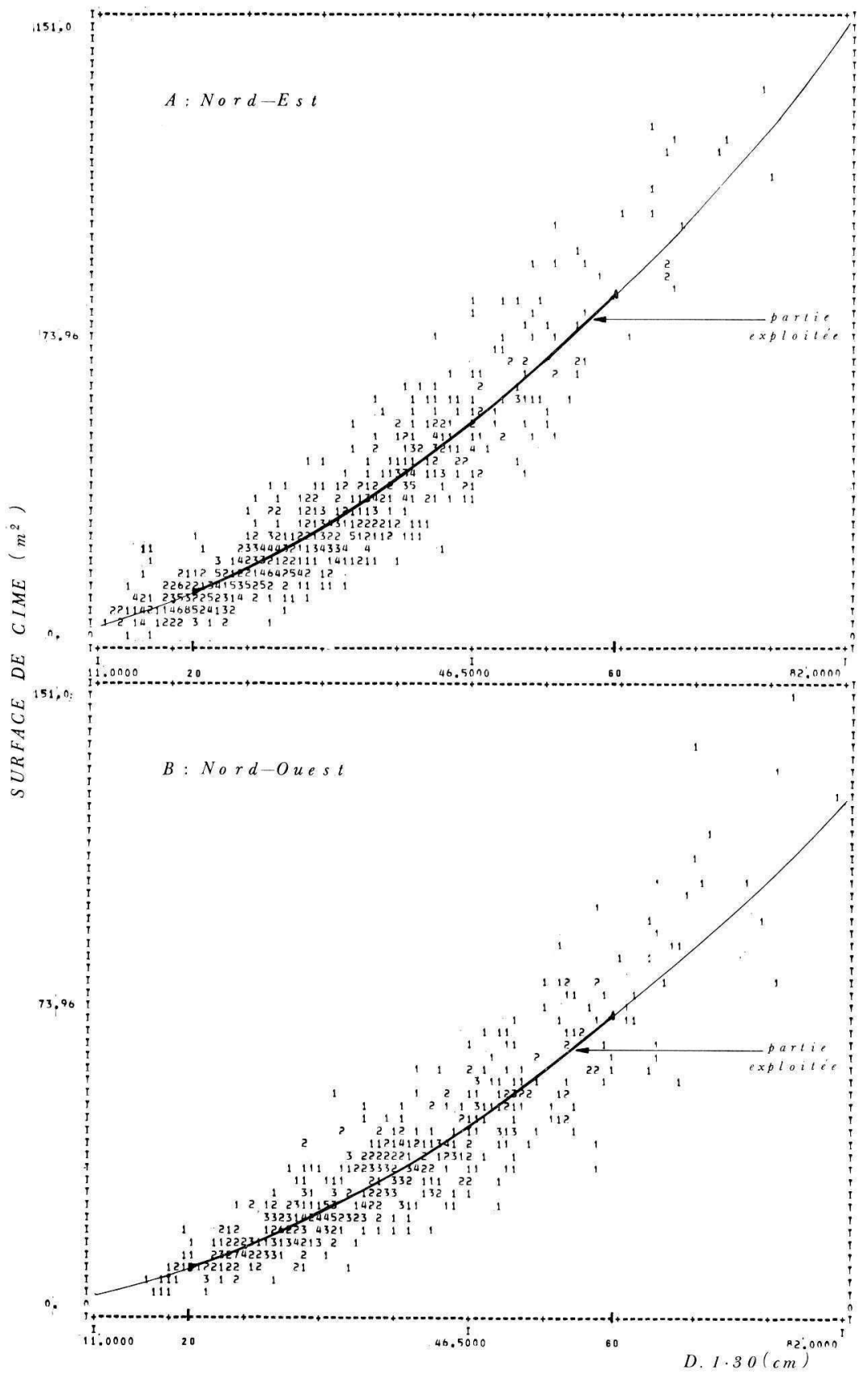

FIG. 6. - Surface de projection de cime en fonction du diamètre à $1,30 \mathrm{~m}$ pour les arbres des hêtraies du N.-E. (A) ef du N.-O. (B).

Scatter diagrams of crown projection area versus $d b h$ for trees of north-east (A) and north-west (B) beech stands. 
Comme nous le verrons, dans la définition d'une mesure de densité suivant les principes de Krajicek et al. (ou de Chisman et Schumacher), la relation finalement utilisée est celle qui donne la surface de couronne en fonction du diamètre à $1,30 \mathrm{~m}$. Les diagrammes de dispersion de la surface de couronne en fonction du diamètre (D) ont donc été examinés pour chaque région (fig. 6). Un premier ajustement sans précautions particulières, d'un binôme (en $D$ et $D^{2}$ ) à ces données par la méthode des moindres carrés a conduit à des résultats incohérents dans les développements ultérieurs. Un examen plus attentif de ces diagrammes montre une dispersion croissante de la surface de cime quand le diamètre augmente. Cet aspect a conduit à postuler que l'écart-type de la distribution de la surface de couronne pour un diamètre donné $D$ est proportionnel à une puissance de ce diamètre. Après quelques tentatives, la valeur 0,7 a été retenue pour les deux régions. En pondérant alors les données de surface de couronne par $\left(1 / D^{0,7}\right)$ on obtient de nouvelles données de dispersion homogène et dont, en outre, on peut rendre comple correctement par une relation linéaire ( ${ }^{1}$ ) (fig. 7). La méthode des moindres carrés appliquée à ces données conduit aux estimations suivantes théoriquement sans biais :

Nord-Est $: \frac{S C}{D^{0,7}}=-0,6346+0,09007 \mathrm{D}, \quad r^{2}=0,847 \quad 639$ observations

Nord-Ouest : $\frac{\mathrm{SC}}{\mathrm{D}^{0,7}}=-0,4095+0,07448 \mathrm{D}, \quad \mathrm{r}^{2}=0,812 \quad 529$ observations

$\mathrm{SC}=$ Surface de couronne en $\mathrm{m}^{2}$

$\mathrm{D}=$ Diamètre à $1,30 \mathrm{~m}$ en $\mathrm{m}$.

D'où :

$$
\begin{aligned}
& \text { N.-E. : }: S C=-0,6346 D^{0,7}+0,09007 D^{1,7} \\
& \text { N.-O. }: S C=-0,4095 D^{0,7}+0,07448 D^{1,7} .
\end{aligned}
$$

Les graphes des relations (3) et (4) sont représentés sur la figure 6.

\section{V. - Application des relations à la définition d'une mesure de densité pour chaque région}

Le principe d'une mesure de densité basée sur une relation du même type que celles - (3) et (4) - déterminées dans le chapitre III est le suivant : étant donné un peuplement fermé, la surface qu'il occuperait s'il avait la densité moyenne de référence est estimée par application de la relation à tous les arbres du peuplement, et par définition sa densité est égale au rapport de la surface ainsi estimée à la surface réelle du peuplement. On mesure de cette façon l'intensité de la concurrence dans un peuplement forestier par ses effets sur les individus dont elle modifie la morphologie (voir fig. 1).

Le terme de CCF qui désignait la mesure de densité définie par Krajicek et al. a été repris. Soient $\left(a_{1}, b_{1}\right),\left(a_{2}, b_{2}\right)$ les coefficients de l'une des relations (4) ou (3) ;

(1) Bien que la méthode informelle employée ici ne soit pas entièrement satisfaisante, elle permet de réduire l'effet de quelques valeurs erratiques observées pour les plus gros diamètres. 


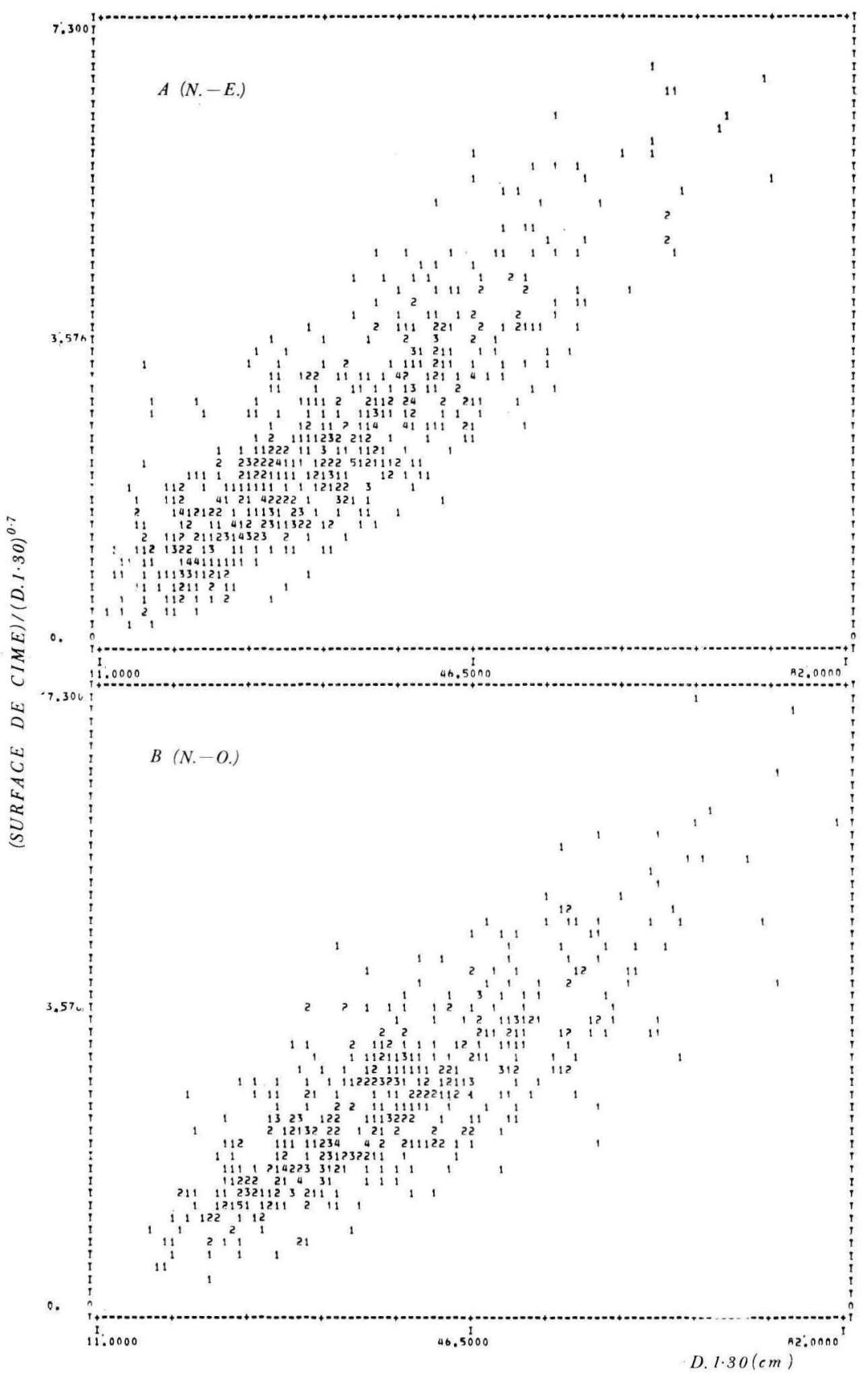

FIG. 7. - Surface de projection de cime rapportée à une puissance du diamètre à $1,30 \mathrm{~m}$ en fonction de ce diamètre (la dispersion est homogène).

Scatter diagrams of ratios of crown projection area to $a \mathrm{dbh}$ power versus $\mathrm{dbh}$ (there is no fendency for the data to spread out with increasing values of dbh). 
la densité (CCF) d'un peuplement fermé de superficie 1 ha dans la région concernée s'écrit, exprimée en pourcentage :

$$
\mathrm{CCF}=100 \frac{1}{10000} \sum_{i=1}^{N}\left(a_{1} D_{i}^{b_{1}}+a_{2} D_{i}^{b_{2}}\right)
$$

la somme étant étendue aux $\mathrm{N}$ arbres dominants et codominants du peuplement :

$$
C C F=\frac{1}{100}\left(a_{1} \sum_{i=1}^{N} D_{i}^{b_{1}}+a_{2} \sum_{i=1}^{N} \frac{D_{i}^{b_{2}}}{2}\right) .
$$

Dans les expressions correspondant aux relations (3) et (4) employées par Gingrich (1967) apparaissaient des termes en $D$ et $D^{2}$ à la place des termes en $D^{b_{1}}$ et $D^{b_{2}}$. Gingrich parvenait alors à une expression finale de la densité d'un peuplement en fonction du nombre de tiges, du coefficient de variation de la distribution des diamètres, et de la surface terrière du peuplement. Malgré les exposants fractionnaires de la relation (5), il a été possible de parvenir à une même expression finale (ces calculs sont détaillés dans l'appendice 2 ) :

$C C F=\frac{1}{100} N\left\{a_{1} D_{G}^{b_{1}}\left(1+\frac{b_{1}\left(b_{1}-2\right)}{2} C V^{2}\right)+a_{2} D_{G}^{b_{2}^{2}}\left(1+\frac{b_{2}\left(b_{2}-2\right)}{2} C V^{2}\right)\right\}$

$\mathrm{CCF}=$ Densité d'un peuplement fermé de surface 1 ha ;

$\mathrm{N}=$ Nombre de tiges des étages dominant et codominant ;

$\mathrm{D}_{\mathrm{G}}=$ Diamètre de l'arbre de surface terrière moyenne du peuplement;

$\mathrm{CV}=$ Coefficient de variation des diamètres des arbres.

(Voir l'appendice 1 pour une définition formelle des termes utilisés.)

Le coefficient de variation des diamètres a été calculé pour un grand nombre de peuplements de hêtre dans les deux régions, non seulement pour des peuplements traités de façon habituelle, mais aussi pour des peuplements faiblement ou fortement éclaircis. L'ensemble des valeurs du coefficient de variation se situe dans l'intervalle allant de 0,15 à 0,30. Un calcul d'erreur effectué sur l'expression (6) montre que dans l'intervalle $(0,15-0,30)$, l'erreur relative faite sur la valeur exacte de la mesure de densité en fixant $C V=0,2$ ne dépasse pas 2 p. 100. Ces considérations permettent de déterminer la densité d'un peuplement donné à partir de sa surface terrière $(G)$ et du nombre de tiges à l'hectare $(\mathrm{N})$. La figure 8 est une représentation graphique (due à Gingrich, 1967) des valeurs de l'expression (6) lorsque $G$ et $N$ varient. Cette figure propose, pour chaque région, des courbes d'isodensité pouvant servir de norme pour le traitement des peuplements de hêtre.

\section{VI. - Les normes de densité}

Les normes de densité de la figure 8 sont des courbes définissant la surface terrière et le nombre de tiges que doivent posséder les peuplements pour avoir une densité donnée, exprimée en pourcentage de la densité moyenne de peuplements traités de façon habituelle. L'ensemble de ces courbes est une représentation imagée des valeurs de l'expression (6) lorsque $\mathrm{G}$ et $\mathrm{N}$ varient ; il vaut mieux le considérer ainsi plutôt 
que d'attacher de l'importance à la continuité de chacune des courbes. Les relations (1) et (2) étant significativement différentes $\left({ }^{1}\right)$, les différences qui apparaissent entre les normes de chaque région (fig. $8 \mathrm{~A}$ et $8 \mathrm{~B}$ ) ne sont très probablement pas dues à l'échantillonnage. Si l'on admet que les densités de référence dans chacune des régions sont sensiblement égales (fig. 4), ces différences peuvent être largement attribuées aux conditions de croissance différentes dans chaque région (fig. 2).

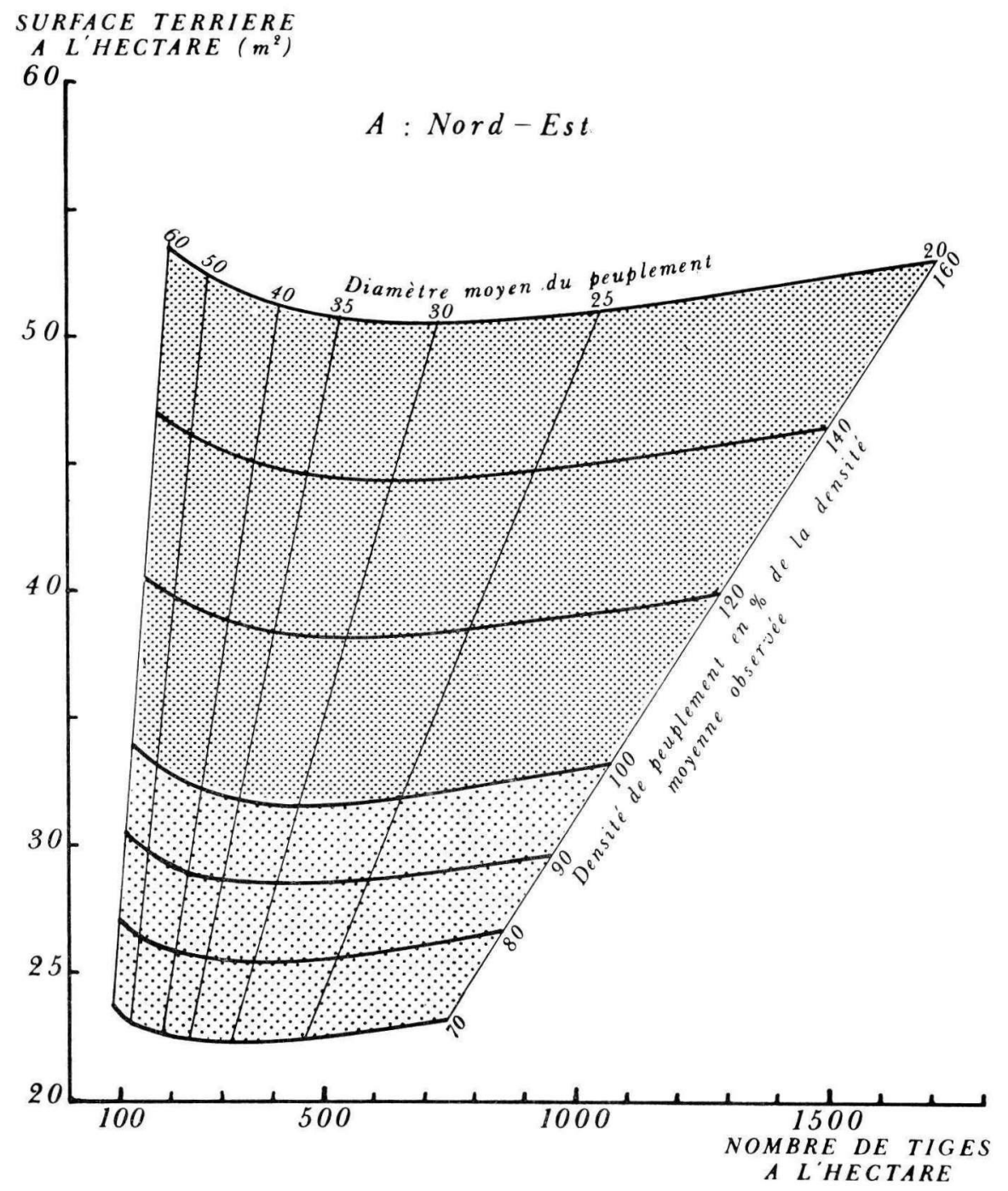

FIG. 8A. - Relation entre la surface terrière, le nombre de tiges, le diamètre moyen et la densité exprimée en p. 100 de la densité moyenne observée pour les peuplements du N.-E. Stocking guides for beech stands in the north-east of France.

(1) Au seuil de 1 p. 100 du test F. 


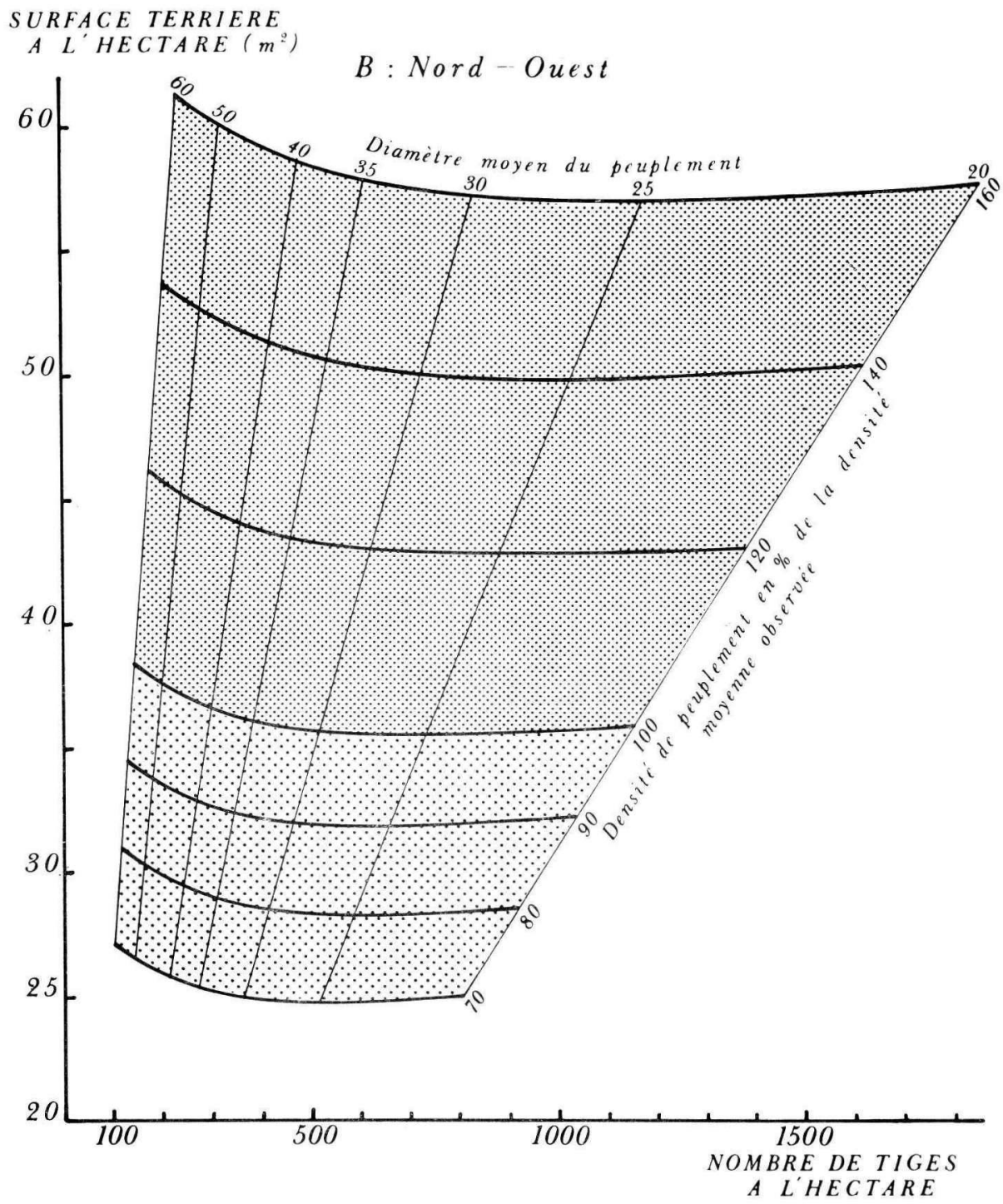

FIG. 8B. - Relation entre la surface terrière, le nombre de tiges, le diamètre moyen et la densité exprimée en p. 100 de la densité moyenne observée pour les peuplements du N.-O.

Stocking guides for beech stands in the north-west of France.

L'intervalle des diamètres moyens utilisé pour les représentations de la figure 8 a été choisi de manière à ne pas déborder celui des diamètres observés et correspond sensiblement à l'intervalle des diamètres moyens des peuplements échantillonnés. L'intervalle des densités correspond à celui des valeurs observées dans les places d'expérience sur les éclaircies du hêtre de la Station de Sylviculture et Production du C.N.R.F. : le niveau 160 est celui de peuplements témoins jamais éclaircis, le niveau 70 
celui de peuplements expérimentaux éclaircis très fortement. L'examen des places d'expérience a procuré l'occasion d'effectuer un test limité de la mesure de densité proposée.

Les courbes d'isodensité de la figure 8 présentent comme celles de Gingrich une concavité dirigée vers le haut. Cependant, la surface terrière n'est pas une fonction décroissante du nombre de tiges comme c'est le cas pour les courbes obtenues par Gingrich pour des peuplements composés de chênes et de noyers ; contrairement à ce qui se passe pour ces peuplements, la surface terrière apparaît ici comme une assez bonne approximation de la mesure de densité pour les peuplements de hêtre du nord de la France.

Les résultats publiés des expériences d'éclaircie européennes sur le hêtre (Braathe, 1957 ; Möller, 1954), bien que parfois contradictoires, donnent à penser que dans l'intervalle des densités retenues la production totale ne devrait pas varier dans des proportions dépassant 10 p. 100. Par contre, à un âge donné, à une densité plus forte correspond une croissance individuelle des arbres plus lente et dans la pratique c'est surtout ce phénomène qu'il faudra considérer, suivant les objectifs poursuivis, lorsque l'on devra décider de laisser la densité augmenter ou bien d'éclaircir pour la diminuer. Si c'est cette dernière opération qui a été choisie, il faudra garder à l'esprit que la nouvelle densité du peuplement ne pourra être précisément évaluée qu'après fermeture du peuplement alors que sa surface terrière aura augmenté ; il faudra donc tenter de tenir compte de cet accroissement de façon anticipée.

Avec une mesure de densité de ce type, les diverses modalités d'éclaircie peuvent prendre tout leur sens. A condition d'intervenir suffisamment tôt dans la vie d'un peuplement, l'écaircie par le bas ou l'éclaircie par le haut (en anglais Crown thinning) correspondent à deux objectifs distincts qui peuvent être bien définis. En effet, en jouant sur la variabilité individuelle des arbres, on peut, pour obtenir une même densité de peuplement, choisir d'avoir un nombre plus ou moins grand de tiges. Le deuxième type d'éclaircie s'oppose au premier par le fait qu'il correspond à la même intensité de concurrence, mais à celle d'une population moins nombreuse d'arbres plus « vigoureux $\gg$.

Les mesures de densité définies par la relation (5) peuvent directement être employées dans des travaux de recherches où il est important de déterminer avec précision les conditions de croissance des arbres. II n'est pas impossible que dans certains cas d'études de croissance, de régénération, ou d'état sanitaire, ces mesures de densité, indépendantes de l'âge et de la station, puissent apporter une information précieuse dans l'analyse et la compréhension de phénomènes comme cela s'est déjà produit avec le CCF aux U.S.A. (Voir Ottorini, 1978).

\section{Conclusion}

A l'issue de cette étude, il apparaît que d'importants travaux réalisés aux U.S.A. sur la mesure des densités des peuplements, conçue pour tenir compte des effets de concurrence sur les individus, ont réussi à être adaptés dans le cas particulier de peuplements réguliers éclaircis. Cela a pu être réalisé sans disposer de peuplements 
normaux $\left({ }^{1}\right)$ ou d'arbres « de plein champ ». Les motivations qui président à la recherche de ces mesures de densité ne sont pas éloignées de celles qui conduisirent Hart dès 1928 à ramener un espacement moyen par arbre à la hauteur des peuplements, car il s'agit toujours de relativiser l'espace vital par la dimension des individus. Cependant, les principes présentés ici permettent d'introduire la struciure des peuplements dans la mesure de densité, ef d'évaluer ainsi avec une meilleure précision l'intensité de la concurrence, procurant une précieuse information qui peut s'avérer ứle dans des recherches de diverses natures concernant les peuplements forestiers.

La figure 8 se présente sous forme d'un ensemble de normes permetiant d'évaluer, pour chacune des deux régions de croissance définies, la densité d'un peuplement donné en fonction de la surface terrière et du nombre de tiges à l'hectare. Ces normes peuvent servir de guide pour contrôler la densité des peuplements de hêtre à partir de caractéristiques usuelies qui sont familières. Cependant, pour permettre d'utiliser effectivement les résultats de cețe étude dans la pratique sylvicole, il reste à franchir une certaine distance nécessitant une réflexion spécifique en tenant compte du fait que la croissance des arbres du peuplement restant sur pied après l'éclaircie est modifiée. Un effort allant dans ce sens est dójà engagé.

Reçu pour publication en février 1979.

\section{Remerciements}

Les calculs et les impressions automatiques de graphiques (en particulier ceux des phases exploratoires qui n'apparaissent pas dans ce texte) ont été effectués à la Station de Biométrie du C.N.R.F. en utilisant sa bibliothèque de programmes.

\section{Summary}

Stocking standards for beech stands in the north-east and the north-west of France

Since 1961 the Crown Competition Factor of Krajicek ef al. which is closely related to the Tree Area Ratio of Chisman and Schumacher - has proved its efficiency as a measure of stand density through numerous applications in the U.S.A. This work is adapted from the paper by S. F. Gingrich on " stocking and stand density in Upland hardwood forests ": here, the constant density of regularly thinned beech stands is used as a density standard by means of the relation between crown-width and d.b.h. of the trees.

Two growth regions have been characterized in the north of France, and random samples of beech stands of various ages in the two best site quality classes of each region have shown a good linear relationship between crown-width and d.b.h. of the trees in the stands in each region. According to the studies of Curtin and Gingrich, this characteristic relationship has been used to ensure a constant density of all stands, and then to construct a density measure following Krajicek et al.

Special attention was necessary for fitting the relations in order to obtain coherent results for the stocking standards in both regions. These standards define those characteristics (basal area and number of stems per ha, mean diameter) which beech stands must follow to grow with a constant density, expressed in percent age of the mean density observed at present in the thinned stands in the North of France.

\section{Références bibliographiques}

BRAATHE P., 1957. Thinning in even-aged stands. (A summary of European literature.) Faculty of Forestry, University of New Brunswick, Fredericton, 1957.

CHISMAN H. H. and SCHUMACHER F. X., 1940. On the tree-area ratio and certain of its applications. Journ. For., 38, 311-317.

(1) Terme employé aux U.S.A. ef au Canada pour désigner des peuplements naturels équiennes de densité maximale. 
CURTIN R. A., 1970. Dynamics of tree and crown structure in Eucalyptus Obliqua. Forest Sci., 16 (3), $321-328$.

CURTIS R. O., 1970. Stand density measures : an interpretation. Forest Sci., 16 (4), 403-414.

CZARNOWSKY, M. S., 1961. Dynamics of even-aged Forest stands. Lovisiana State University Press.

DREW T. J. and FLEWELLING J. W., 1977. Some recent Japanese theories of yield-density relationships and their application to Monterey pine plantations. Forest Sci., 23 (4), 517-534.

GINGRICH S. F., 1967. Measuring and evaluating stocking and stand density in Upland hardwood Forests in the Central States. Forest Sci., 13 (1), 38-53.

HAMILTON G. J. and CHRISTIE J. M., 1971. Forest Management Tables (Metric). Her Majesty's Stationery Office.

HUMMEL F. C., 1954. The definition of thinning treatments. Proceedings of the 2 nd Congress of I.U.F.R.O., Rome, 1953, 582-588.

KRAJICEK J. E., BRINKMAN K. A. and GINGRICH S. F., 1961. Crown competition, a measure of density. Forest Sci., 7 (1), 35-42.

LE GOFF N., 1974. La croissance du hêtre en France. Utilisation possible de tables de production étrangères pour suivre l'évolution des peuplements. Document interne. Station de Sylviculture et Production, C.N.R.F., $n^{\circ} 74 / 3$.

MÖLLER, CARL MAR, 1954. The influence of thinning on volume increment. I. Results of investigations, in : Thinning problems and practices in Denmark. State University of New York. College of Forestry at Syracuse. World Forestry Series Bulletin, $n^{0} 1$.

OTTORINI J. M., 1978. Aspects de la notion de densité et croissance des arbres en peuplement. Ann. Sci. Forest. Vol. 35, 4, 299-320.

PARDÉ J., 1959. Retour sur l'indice C.V.P. de Paterson. Revue Forestière Française, no 1, pp. 50-53.

PARDÉ J., 1961. Comment préciser l'intensité d'une éclaircie. Revue Forestière Française, août-sept. 1961, pp. 551-557.

SCHOBER R., 1971. Die Rotbuche. J. D. Sauerländer's Verlag. Frankfurt am Main.

WILLEY R. W. and HEATH, S. B., 1969. The quantitative relationships between plant population and crop yield. Adv. Agric. 21, 281-321.

\section{Appendice 1 : définitions et symboles}

$\mathrm{H}_{0} \quad$ : hauteur dominante d'un peuplement, moyenne des hauteurs des cent plus gros arbres à l'hectare (en $\mathrm{m}$ ). Elle est liée à la productivité de la station.

N : Nombre de tiges d'un peuplement (ici des étages dominant et codominant du peuplement).

$D_{1,30}$ (ou $D$ ) : Diamètre de la section de la tige d'un arbre mesuré à 1,30 $\mathrm{m}$ de haut (en $\mathrm{cm}$ ).

G : Surface terrière d'un peuplement : somme des surfaces de section transversale à $1,30 \mathrm{~m}$ des tiges du peuplement :

$$
\mathrm{G}=\frac{\pi}{4} \sum_{\mathrm{i}=1}^{\mathrm{N}} \mathrm{D}_{\mathrm{i}}^{2} \quad\left(\text { en } \mathrm{m}^{2}\right) \text {. }
$$

$D_{G} \quad$ : Diamètre de l'arbre de surface terrière moyenne d'un peuplement ; il est tel que :

$$
G=N \frac{\pi}{4} D_{G}^{2} \quad(\text { en } m) .
$$


CV

: Coefficient de variation des diamètres des arbres d'un peuplement

$$
C V^{2}=\frac{\sum_{i=1}^{N}\left(D_{i}-\bar{D}\right)^{2} / N}{\overline{D^{2}}}
$$

ou $N \bar{D}=\sum_{i=1}^{N} D_{i}$ (sans unité).

SC : Surface de couronne d'un arbre (définie dans le texte) (en $\mathrm{m}^{2}$ ).

$\mathrm{HC} / \mathrm{HT}$ : Rapport de la hauteur de cime à la hauteur totale d'un arbre. Pour un peuplement, c'est la moyenne de ces valeurs.

\section{Appendice 2 : détail des calculs conduisant à la relation (6)}

II s'agit de montrer comment la relation (5) peut être reformulée dans les termes de la relation (6).

Les calculs sont développés sur les monomes de la forme $y_{i}=a D_{i}^{b}$ où les $D_{i}$ représentent les $\mathrm{N}$ diamètres des arbres de l'étage dominant d'un peuplement forestier. En posant $e_{i}=D_{i}-\bar{D}$, il vient :

$$
\sum y_{i}=a \sum\left(e_{i}+\bar{D}\right)^{b}=a \bar{D}^{b} \sum\left(1+\frac{e_{i}}{\bar{D}}\right)^{b} .
$$

Un développement limité donne alors:

$$
\sum y_{i} \simeq a \bar{D}^{b}\left(N+\frac{b}{\bar{D}} \sum e_{i}+\frac{b(b-1)}{2} \frac{\sum e_{i}^{2}}{\bar{D}^{2}}\right)
$$

d'où :

$$
\sum y_{i} \simeq N a \bar{D}^{b}\left(1+\frac{b(b-1)}{2} C V^{2}\right)
$$

Plusieurs calculs ont pu montrer que les différences entre la valeur exacte de $\sum y_{i}$ et sa valeur approchée étaient insignifiantes pour les peuplements ayant permis ce test. Comme d'autre part :

$$
\overline{\mathrm{D}}=\frac{\mathrm{D}_{\mathrm{G}}}{\sqrt{1+\mathrm{CV}^{2}}}
$$

un nouveau développement limité conduit à l'expression voulue :

$$
\sum y_{i} \simeq N a D_{G}^{b}\left(1+\frac{b(b-2)}{2} C V^{2}\right)
$$

